\title{
Impact of moderate versus mild aerobic exercise training on inflammatory cytokines in obese type 2 diabetic patients: a randomized clinical trial
}

\author{
*Abd El- Kader SM SM $^{1}$ Gari AM², Salah El-Den AEM ${ }^{1}$
}

1. Department of Physical therapy, Faculty of Applied Medical Sciences, King Abdulaziz University, Saudi Arabia

2. Department of Medical Laboratory Technology, Faculty of Applied Medical Sciences, King Abdulaziz University, Saudi Arabia.

\begin{abstract}
Background: Recently some plasma biomarkers of inflammation have been recognized as important cardiovascular risk factors. There is little information about the effects of aerobic exercise training on these biomarkers and the risk of metabolic complications in obese type 2 diabetes patients.

Objective: To compare the impact of moderate versus mild aerobic exercise training on the inflammatory cytokines in obese type 2 diabetic patients.

Methods: Fifty obese type 2 diabetic patients of both sexes with body mass index (BMI) varying from 31 to $36 \mathrm{~kg} / \mathrm{m}^{2}$, non smokers, free from respiratory, kidney, liver, metabolic and neurological disorders, participated in this study. Their age ranged from 40 to 55 years. The subjects were included into two equal groups; the first group (A) received moderate aerobic exercise training. The second group (B) received mild aerobic exercise training, three times / week for 3 months. Results: The mean values of leptin, TNF- alpha, IL2, IL4, IL6, HOMA-IR and HBA1c were significantly decreased in group (A) and group (B). Also, there were significant differences between both groups after treatment.

Conclusion: Moderate aerobic exercise training modulates inflammatory cytokines more than mild aerobic exercise training in obese type 2 diabetic patients.
\end{abstract}

Key words: Aerobic exercise, inflammatory cytokines, obesity, non-insulin dependent diabetes African Health Sciences 2013; 13(4): 857 - 863 http://dx.doi.org/10.4314/ahs.v13i4.1

\section{Introduction}

Type 2 diabetes is one of the fastest growing public health problems. Cardiovascular disease (CVD), the number one cause of mortality in the USA, is almost twice as common as in individuals with diabetes ${ }^{1}$. Morbidity and mortality in type 2 diabetes is mainly associated with atherosclerotic cardiovascular disease and late complications as a result of dysfunction of plasma biomarkers of inflammation including leptin, tumor necrosis factor- alpha (TNF-alpha) and interleukin-6 (IL-6). These factors have paracrine/ autocrine functions that include regulation of energy expenditure, in part, by modulating whole-body insulin sensitivity ${ }^{2}$. Tumor necrosis factor alpha

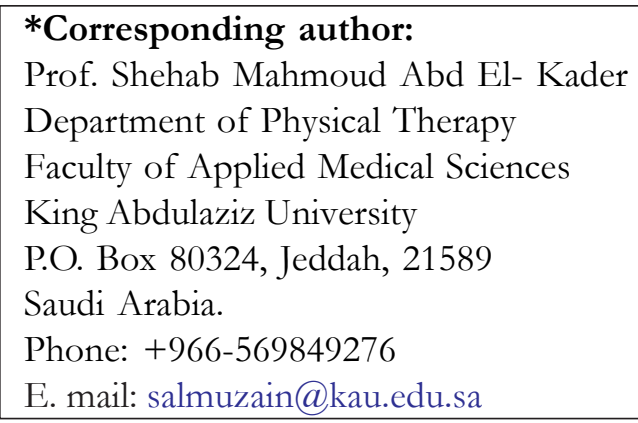

(TNF-alpha) induced insulin resistance by interacting with insulin receptor signaling and through activation of lipolysis and inhibition of lipoprotein lipase. TNF-alpha was proposed to have preferentially paracrine effects and to be a regulator of insulin resistance at the tissue level ${ }^{3-5}$.

Low-grade inflammation is a common feature in subjects with type 2 diabetes $^{6}$ as increased levels of circulating inflammatory markers were found to be significantly more prominent in type 2 diabetes ${ }^{7}$. Increased inflammatory markers in type 2 diabetes are believed to originate, at least partially, from adipose tissue ${ }^{8,9}$.

Interleukin (IL)- 6 and tumor necrosis factor alpha (TNF-alpha) are two major pro-inflammatory cytokines, secreted in significant amounts from adipose tissue. Consequently obese women (healthy and diabetic) have higher cytokine levels than healthy lean women. Furthermore, increased levels of IL-6 and TNF-alpha are associated with deterioration of glycemic control, increased IR, and dyslipidemia, contributing to the dysfunctional metabolic status of obese and type 2 diabetic individuals ${ }^{10}$. Adipose tissue 
is an active endocrine tissue, which secretes hormones, such as adiponectin, resistin, and leptin appear to contribute to inflammation, atherosclerosis, and may be involved in the etiology of type 2 diabetes, possibly constituting the missing link between obesity and insulin resistance ${ }^{11}$. Leptin alters metabolism by mediating appetite and energy expenditure via feedback mechanism on the hypothalamic satiety-regulating centers and has also been linked to obesity-related IR. Leptin resistance is associated with the development of insulin resistance in individuals with type 2 diabetes, and in animals, administration of leptin reverses insulin resistance ${ }^{12}$.

Exercise improves diabetic status and reduces the metabolic risk factors associated with cardiovascular diseases and improves insulin sensitivity ${ }^{13}$. Regular exercise increases maximum oxygen uptake, reduces insulin resistance, reduces obesity and prevents further weight gain, and dampens the inflammatory markers, suggesting that habitual exercise plays an important role in the prevention and treatment of obesity, insulin resistance, and coronary heart disease ${ }^{14}$. Few studies have prospectively examined the effect of exercise on elevated levels of inflammatory biomarkers in diabetic subjects and found contrasting results in terms of efficacy and dependence on weight loss ${ }^{15,16}$. However, combined exercise has greater antiinflammatory effects than aerobic or resistance exercise alone causing a deepest decrease in CRP, IL-6, TNF alpha, leptin, and resistin and a higher increase in anti-inflammatory cytokines such as interleukin-4 (IL-4), interleukin-10 (IL-10), and adiponectin ${ }^{17}$. Moreover, the type, dose and intensity of physical activity needed to obtain a significant anti-inflammatory effect in this high-risk population are largely unknown.

Aerobic exercise training prevents the development of chronic inflammation associated with NIDDM by reducing the production of proinflammatory cytokines TNF- alpha and IL-6. The beneficial effects of regular physical activity include improving insulin resistance, dyslipidemia, and levels of pro-inflammatory cytokines ${ }^{18}$. The potential mechanisms for the anti-inflammatory effect of exercise, include reduced percentage of body fat and macrophage accumulation in adipose tissue, musclereleased interleukin-6 inhibition of TNF-alpha, and the cholinergic anti-inflammatory pathway ${ }^{19}$.

Circulating inflammatory biomarkers originate from multiple sources, particularly visceral adipose tissue, where excess fat promotes macrophage recruitment and both adipocytes and macrophages secrete numerous cytokines (adipokines), including IL-6, TNF- alpha and leptin ${ }^{4}$ . Skeletal muscle is another source of cytokines, called myokines, among which IL-6 is considered to play a dual role as anti-inflammatory and proinflammatory. High-intensity training of long duration may significantly influence myokine production, thus driving the anti-inflammatory effect of exercise ${ }^{20}$.

The aim of this study was to compare the impact of moderate versus mild aerobic exercise training on the inflammatory cytokines in obese type 2 diabetic patients.

\section{Methods}

\section{Subjects}

Fifty obese type 2 diabetic patients of both sexes with body mass index (BMI) ranging from 31 to 36 $\mathrm{kg} / \mathrm{m}^{2}$, non smokers, free from respiratory, kidney,liver, metabolic and neurological disorders participated in this study. Their age ranged from 40 to 55 years. The subjects were included into 2 equal groups: the first group (A) received moderate aerobic exercise training, while the second group (B) received mild aerobic exercise training three times a week for 3 months. Informed consent was obtained from all participants.

This study was approved by the Scientific Research Ethical Committee, Faculty of Applied Medical Sciences at King Abdulaziz University. All participants were free to withdraw from the study at any time. If any adverse effects had occurred, the experiment would be terminated and the Human Subjects Review Board would be informed. However, no adverse effects occurred, and so the data of all the participants were available for analysis.

\section{Evaluated parameters Chemical analysis:}

Blood sample after fasting for 12 hours was taken from each patient in a clean tube containing afew $\mathrm{mg}$ of K2EDTA, centrifuged and plasma was separated and stored frozen at $-20^{\circ}$ to be used for estimation of leptin, plasma TNF-alpha, $\mathrm{C}$ - reactive protein (CRP), interleukin-2 (IL-2), interleukin-4(IL4), interleukin-6 (IL-6) and glycosylated hemoglobin $\left(\mathrm{HBA}_{1} \mathrm{c}\right)$ using colorimetric method. Homeostasis Model Assessment-Insulin Resistance (HOMA) index for insulin sensitivity was computed following this equation: [fasting glycemia $(\mathrm{mmol} / \mathrm{L}) \cdot$ fasting insulin $(\mathrm{mIU} / \mathrm{L})] / 22.5^{21}$. 


\section{The aerobic exercise training program}

The aerobic treadmill-based training program (Track master 400E, gas fitness system, England) was at 65 $\%$ to $75 \%$ of the maximum heart rate (HRmax) achieved according to a modified Bruce protocol for group (A) who received moderate intensity aerobic exercise training, where as group (B) received mild intensity aerobic exercise training at $55 \%$ to $65 \%$ of the maximum heart rate (HRmax) .This rate was defined as the training heart rate (THR). After an initial, 5-minute warm-up phase performed on the treadmill at a low load, each endurance training session lasted 30 minutes and ended with 5-minute recovery and relaxation phase. All patients performed three sessions / week (i.e. a total of 36 sessions per patient over a 3month period).

\section{Statistical analysis}

Table 1: Mean value and significance of Leptin, TNF- alpha, IL-2, IL-4, IL-6, HOMA-IR and $\mathrm{HBA}_{1} \mathrm{c}$ in group (A) before and after treatment

\begin{tabular}{|c|c|c|c|c|}
\hline & \multicolumn{2}{|c|}{ Mean \pm SD } & \multirow{2}{*}{$\begin{array}{c}\text { T- } \\
\text { value }\end{array}$} & \multirow{2}{*}{$\begin{array}{c}\text { P- } \\
\text { value }\end{array}$} \\
\hline & Before & After & & \\
\hline Leptin $(\mathrm{Ng} / \mathrm{ml})$ & 6.5340 .8 & $34.65 \pm 5.42$ & 8.41 & 0.003 \\
\hline TNF- $\alpha(\mathrm{pg} / \mathrm{mL})$ & $5.52 \pm 1.98$ & $4.35 \pm 1.26$ & 6.72 & 0.007 \\
\hline $\mathrm{IL}-2(\mathrm{pg} / \mathrm{mL})$ & $7.98 \pm 2.25$ & $4.43 \pm 1.86$ & 5.78 & 0.008 \\
\hline $\mathrm{IL}-4(\mathrm{pg} / \mathrm{mL})$ & $5.43 \pm 1.62$ & $3.45 \pm 1.78$ & 5.12 & 0.009 \\
\hline IL-6 (pg/mL) & $8.89 \pm 2.13$ & $5.22 \pm 1.69$ & 6.33 & 0.006 \\
\hline $\mathrm{HBA}_{1} \mathrm{c}(\%)$ & $7.98 \pm 2.56$ & $6.13 \pm 1.55$ & 6.11 & 0.005 \\
\hline HOMA-IR & $4.73 \pm 1.28$ & $2.85 \pm 1.16$ & 6.42 & 0.008 \\
\hline
\end{tabular}

TNF-alpha $=$ tumor necrosis factor - alpha. $\quad$ IL-2 $=$ Interleukin- 2 IL-4 $=$ Interleukin- 4

IL-6 = Interleukin-6 HBA1c = glycosylated hemoglobin. HOMA-IR = Homeostasis Model Assessment-Insulin Resistance (HOMA-IR) index

Table 2: Mean value and significance of Leptin, TNF- alpha,, IL-2, IL-4, IL-6, HOMA-IR and $\mathrm{HBA}_{1} \mathrm{c}$ in group (B) before and after treatment

\begin{tabular}{lrrlrr}
\hline & \multicolumn{2}{c}{ Mean $\underline{\mathbf{+} S D}$} & \multirow{2}{*}{ T- } & P-value \\
& \multicolumn{1}{c}{ value } & \\
\cline { 2 - 4 } & \multicolumn{1}{c}{ Before } & \multicolumn{1}{c}{ After } & & \\
\hline Leptin $\mathrm{Ng} / \mathrm{ml} \pm 6.62$ & $37.34 \pm 5.91$ & 4.11 & 0.012 \\
TNF- $\alpha(\mathrm{pg} / \mathrm{mL})$ & $5.71 \pm 1.85$ & $5.01 \pm 1.42$ & 3.13 & 0.026 \\
$\mathrm{IL}-2(\mathrm{pg} / \mathrm{mL})$ & $7.88 \pm 2.52$ & $6.37 \pm 2.16$ & 3.24 & 0.018 \\
$\mathrm{IL}-4(\mathrm{pg} / \mathrm{mL})$ & $5.77 \pm 1.84$ & $4.51 \pm 1.42$ & 3.21 & 0.025 \\
$\mathrm{IL}-6(\mathrm{pg} / \mathrm{mL})$ & $8.96 \pm 2.53$ & $6.88 \pm 2.01$ & 3.19 & 0.013 \\
$\mathrm{HBA}_{1} \mathrm{c}(\%)$ & $7.78 \pm 2.66$ & $6.93 \pm 1.85$ & 3.14 & 0.018 \\
HOMA-IR & $4.94 \pm 1.75$ & $3.92 \pm 1.46$ & 3.28 & 0.015 \\
\hline
\end{tabular}

TNF- alpha $=$ tumor necrosis factor - alpha. $\quad$ IL-2 $=$ Interleukin-2 IL-6 = Interleukin- $6 \quad$ HBA1c $=$ glycosylated hemoglobin.

HOMA-IR = Homeostasis Model Assessment-Insulin Resistance (HOMA-IR) index 
Table 3: Mean value and significance of Leptin, TNF- alpha,, IL-2, IL-4, IL-6, HOMA-IR and HBA $_{1} \mathrm{c}$ in group (A) and group (B) after treatment

\begin{tabular}{|c|c|c|c|c|}
\hline & \multicolumn{2}{|c|}{ Mean + SD } & \multirow[t]{2}{*}{ T-value } & \multirow[t]{2}{*}{ P-value } \\
\hline & Group (A) & Group (B) & & \\
\hline Leptin $\mathrm{Ng} / \mathrm{ml}$ & $34.65 \quad 5.42$ & $37.34 \pm 5.91$ & 4.23 & 0.012 \\
\hline TNF- $\alpha(\mathrm{pg} / \mathrm{mL})$ & $4.35 \pm 1.26$ & $5.01 \pm 1.42$ & 3.52 & 0.026 \\
\hline $\mathrm{IL}-2(\mathrm{pg} / \mathrm{mL})$ & $4.43 \pm 1.86$ & $6.37 \pm 2.16$ & 3.14 & 0.018 \\
\hline $\mathrm{IL}-4(\mathrm{pg} / \mathrm{mL})$ & $3.45 \pm 1.78$ & $4.51 \pm 1.42$ & 3.12 & 0.025 \\
\hline IL-6 (pg/mL) & $5.22 \pm 1.69$ & $6.88 \pm 2.01$ & 3.41 & 0.013 \\
\hline $\mathrm{HBA}_{1} \mathrm{c}(\%)$ & $6.13 \pm 1.55$ & $6.93 \pm 1.85$ & 3.16 & 0.038 \\
\hline HOMA-IR & $2.85 \pm 1.16$ & $3.92 \pm 1.46$ & 3.24 & 0.025 \\
\hline
\end{tabular}

TNF- alpha, $=$ tumor necrosis factor - alpha. IL-2 $=$ Interleukin-2 IL-4 = Interleukin- 4

IL-6 = Interleukin-6 HBA1c = glycosylated hemoglobin.

HOMA-IR = Homeostasis Model Assessment-Insulin Resistance (HOMA-IR) index

\section{Discussion}

Even mild physical exercise, that does not affects $\mathrm{VO}_{2} \max$, can cause improvement in the in vivo insulin sensitivity if continued for a prolonged period of time. Aerobic exercise training has traditionally been advocated as an essential component in the medical management of type 2 diabetic patients in order to improve the cardiovascular health of these individuals ${ }^{22}$. Regular aerobic exercise decreases cardiovascular risk of type 2 diabetic patients principally by reducing body weight and abdominal visceral fat accumulation with subsequent improvements in insulin sensitivity, blood pressure, lipid profile and glycaemic control ${ }^{23}$.

There is controversy surrounding the beneficial effects of different aerobic exercise intensities on glycemic control and inflammatory cytokines in obese type 2 diabetes patients, so this study compared the impact of moderate versus mild aerobic exercise training on insulin resistance and inflammatory cytokines in obese type 2 diabetic patients. The mean values of leptin, TNF-alpha, CRP, IL-2, IL-4, IL-6, HOMA-IR and HBA1c were significantly decreased in group (A) which received moderate intensity aerobic exercise training and group (B) which received mild intensity aerobic exercise training. There was a significant difference between both groups after treatment, these findings are supported and confirmed by several previous studies.

A twice-weekly, 6-month, moderate intensity aerobic-training program, without a concomitant weight loss diet, is associated with significant decreases in circulating P-selectin and ICAM-1 levels and with a less atherogenic lipid profile in overweight, non-smoking, older type 2 diabetic individuals ${ }^{24}$.
Eighty-two patients were randomized into 4 groups: sedentary control (A) received lowintensity aerobic exercise, group (B) performed high-intensity aerobic, group $(\mathrm{C})$ performed aerobic and resistance exercises and group (D) performed exercise (with the same caloric expenditure) for 12 months. Physical exercise in type 2 diabetic patients with the metabolic syndrome is associated with a significant reduction of inflammatory and insulin resistance biomarkers, independent of weight loss. Long-term high-intensity (preferably mixed) training, in addition to daytime physical activity, is required to obtain a significant anti-inflammatory effect ${ }^{25}$.

It is possible that aerobic exercise decreases subclinical, chronic inflammation and improves endothelial function simply as a result of reducing obesity (particularly visceral obesity) and improving insulin sensitivity ${ }^{26}$. Most published studies showing beneficial effects of moderate aerobic exercise on insulin sensitivity in obese patients have also constantly reported significant reductions in abdominal visceral fat, thus reinforcing the importance of diminished visceral adipose tissue in the treatment of insulin resistance ${ }^{27,28}$.

During regular aerobic exercise, skeletal muscle fibers inhibit the production of the proinflammatory cytokine TNF-alpha, and produce several anti-inflammatory cytokines (termed "myokines") that may be involved in mediating the health-beneficial effects of exercise ${ }^{29}$. Additionally, exercise training may reduce mononuclear cell production of pro-inflammatory cytokines by reducing chronic oxidative stress ${ }^{30}$. Exercise has the potential to lower the inflammatory status by reduction of high-sensitivity C-reactive protein (hs- 
CRP) and tumor necrosis factor (TNF-alpha) and enhancement of adiponectin ${ }^{31-33}$.

Exercise suppresses the production of proinflammatory cytokines and enhances antiinflammatory cytokines. Because pro-inflammatory cytokines, IL-6 and TNF-alpha, have cytotoxic action, it can be proposed that regular exercise prevents further damage to insulin-producing $\hat{a}$-cells by attenuation of their production ${ }^{34}$. Aerobic exercise decreases subclinical, chronic inflammation and improves endothelial function simply as a result of reducing obesity (particularly visceral obesity) and improving insulin sensitivity ${ }^{35}$.

Several studies suggest that training programs which involve a resistive exercise component, that is moderate intensity weight-lifting exercises, may be of particular benefit in type 2 diabetes as increased muscle mass have been associated with benefits in terms of glycemic control as skeletal muscle represents the largest mass of insulin-sensitive tissue ${ }^{36-38}$.

High-intensity exercise training program induced an improvement of biomarkers of inflammation and insulin resistance, with a reduction of IL-6, TNF- alpha, and leptin (associated with decreased insulin, C-peptide, and HOMA-IR) and an increase of IL-4 and IL-10 thus indicating that exercise has a full anti-inflammatory and insulinsensitizing effect. These results suggest that the beneficial effect of physical activity on CVD morbidity and mortality may depend up on the antiinflammatory effect of exercise, though it is unproven that reducing CRP and other inflammatory biomarkers is effective in decreasing CVD risk. Changes in inflammatory biomarkers in the highintensity exercise were paralleled by improvements, though, to a lesser extent, in $\mathrm{HbA}_{1 \mathrm{c}}{ }^{4}$.

One study has shown that low- to moderate-intensity exercise training is as effective as moderate- to highintensity exercise training ${ }^{39}$. Another study reported that high-intensity training was more effective in improving glycemic control ${ }^{40}$.

\section{Conclusion}

Moderate aerobic exercise training modulates inflammatory cytokines more than mild aerobic exercise training in obese type 2 diabetic patients.

\section{Acknowledgment}

This project was funded by the Deanship of Scientific Research (DSR), King Abdulaziz University, Jeddah, under grant no. (3/142-1431). The authors, therefore, acknowledge with thanks DSR technical and financial support.

\section{References}

1. Kelley GA, Kelley KS. Effects of aerobic exercise on lipids and lipoproteins in adults with type 2 diabetes: A meta-analysis of randomizedcontrolled trials. Public Health 2007; 121:643-655.

2. Neto J, Lira F, Oyama L, Zanchi N, Yamashita A, Batista M. et al. Exhaustive exercise causes an anti-inflammatory effect in skeletal muscle and a pro-inflammatory effect in adipose tissue in rats. Eur J Appl Physiol 2009; 106:697-704.

3. Ruan $\mathrm{H}$, Lodish $\mathrm{H}$. Insulin resistance in adipose tissue: direct and indirect effects of tumor necrosis factor-alpha. Cytokine Growth Factor Rev 2003; 14:447-455.

4. Calabro P, Chang D, Willerson J, Yeh E. Release of C-reactive protein in response to inflammatory cytokines by human adipocytes: linking obesity to vascular inflammation. $J A m$ Coll Cardiol 2005; 46:1112-1113.

5. Tuomisto K, Jousilahti P, Sundvall J, Pajunen P, Salomaa V. C-reactive protein, interleukin-6 and tumor necrosis factor alpha as predictors of incident coronary and cardiovascular events and total mortality. A population-based, prospective study. Thromb Haemost 2006; 95: 511-518.

6. Calle MC, Fernandez ML. Inflammation and type 2 diabetes. Diabetes \& Metabolism 2012; 38: 183-191.

7. Pham MN, Hawa MI, Pfleger C, Roden M, Schernthaner G, Pozzilli P, Buzzetti R, et al. Proand anti-inflammatory cytokines in latent autoimmune diabetes in adults, type 1 and type 2 diabetes patients: action LADA 4. Diabetologia 2011; 54:1630-1638.

8. Mraz M, Lacinova Z, Drapalova J, Haluzikova D, Horinek A, Matoulek M, Trachta P et al. The effect of very-low-calorie diet on mRNA expression of inflammation-related genes in subcutaneous adipose tissue and peripheral monocytes of obese patients with type 2 diabetes mellitus. J. Clin. Endocrinol. Metab. 2011; 96: E606-E613.

9. Sam S, Haffner S, Davidson MH, D'Agostino RB, Feinstein S, Kondos G, Perez A, Mazzone T. Relation of abdominal fat depots to systemic markers of inflammation in type 2 diabetes. Diabetes Care 2009; 32: 932-937. 
10. Pradhan A, Ridker L. Do atherosclerosis and type 2 diabetes share a common inflammatory basis?. Eur Heart J 2002; 23:831-4.

11. Havel P. Control of energy homeostasis and insulin action by adipocyte hormones: leptin, acylation stimulating protein, and adiponectin. Curr Opin Lipidol 2002; 13:51-9.

12. Taylor S, Barr V. and Reitman M. Does leptin contribute to diabetes caused by obesity?. Science 1996; 274:1185-8.

13. Bassuk S, Manson J. Epidemiological evidence for the role of physical activity in reducing risk of type 2 diabetes and cardiovascular disease $J$ Appl Physiol 2005; 99:1193-1204.

14. Kondo N, Nomura M, Nakaya Y, Ito S, Ohguro T. Association of inflammatory marker and highly sensitive $\mathrm{C}$-reactive protein with aerobic exercise capacity, maximum oxygen uptake and insulin resistance in healthy middle-aged volunteers. Circ J 2005; 69: 452-457.

15. Oberbach A, Tönjes A, Klöting N, Fasshauer M, Kratzsch J, Busse M. et al. Effect of a 4 week physical training program on plasma concentrations of inflammatory markers in patients with abnormal glucose tolerance. Eur J Endocrinol 2006; 154:577-585.

16. Zoppini G, Targher G, Zamboni C, Venturi C, Cacciatori V, Moghetti P. et al. Effects of moderate-intensity exercise training on plasma biomarkers of inflammation and endothelial dysfunction in older patients with type 2 diabetes. NutrMetab Cardiovasc Dis Oberbach 2006; 16: 543549.

17. Hopps E, Canino B, Caimi G. Effects of exercise on inflammation markers in type 2 diabetic subjects. Acta Diabetol 2011; 48:183-9.

18. Pedersen B, Toft A. Effects of exercise on lymphocytes and cytokines. BrJ Sports Med2000; 34:246-251.

19. Woods J, Vieira V, Todd K. Exercise, Inflammation, and Innate Immunity. Immunology and Allergy Clinics of North America 2009; 29:381393.

20. Wilund R. Is the anti-inflammatory effect of regular exercise responsible for reduced cardiovascular disease? . Clin Sci 2007; 112: 543555.

21. Robergs RA, Landwehr R. The surprising history of the "HRmax $=220$-age" equation. $J$ Exerc Physiol Online 2002; 5 (2): 1-10.

22. Stewart KJ. Role of exercise training on cardiovascular disease in persons who have type
2 diabetes and hypertension. Cardiol Clin 2004; 22:569-586.

23. Ibanez J, Izquierdo M, Arguelles I, Forga L, Larriòn JL, Garcìa-Unciti M. Twice-weekly progressive resistance training decreases abdominal fat and improves insulin sensitivity in older men with type 2 diabetes. Diabetes Care 2005; 28: 662-667.

24. Zoppini G, Targher G, Zamboni C, Venturi C, Cacciatori V, Moghetti P, Muggeo M. Effects of moderate-intensity exercise training on plasma biomarkers of inflammation and endothelial dysfunction in older patients with type 2 diabetes . Nutrition. Metabolism and Cardiovascular Diseases 2006; 16:543-549

25. Balducci S, Zanuso S, Nicolucci A, Fernando F, Cavallo S, Cardelli P, Fallucca S. Antiinflammatory effect of exercise training in subjects with type 2 diabetes and the metabolic syndrome is dependent on exercise modalities and independent of weight loss. Metabolism and Cardiovascular Diseases 2010; 20: 608-617.

26. Hu G, Jousilahti P, Barengo N, Qiao Q, Lakka T, Tuomilehto J. Physical activity, cardiovascular risk factors, and mortality among Finnish adults with diabetes. Diabetes Care 2005; 28:799-805.

27. Rice B, Janssen I, Hudson R, Ross R. Effects of aerobic or resistance exercise and/or diet on glucose tolerance and plasma insulin levels in obese men. Diabetes Care 1999; 22: 684-691.

28. Giannopoulou I, Fernhall B, Carhart R, Weinstock R, Figueroa A. Effects of diet and/ or exercise on the adipocytokine and inflammatory cytokine levels of postmenopausal women with type 2 diabetes. Metabolism 2005; 54:866-875.

29. Petersen AM, Pedersen BK. The antiinflammatory effect of exercise. J Appl Physiol 2005; 98: 1154-1162.

30. Stewart KJ. Role of exercise training on cardiovascular disease in persons who have type 2 diabetes and hypertension. Cardiol Clin 2004; 22: 569-586.

31. Mathur N. and Pedersen BK. Exercise as a mean to control low-grade systemic inflammation. Mediators Inflamm 2008; 109502.

32. Monzillo LU., Hamdy O. and Horton ES. Effect of lifestyle modification on adipokine levels in obese subjects with insulin resistance. Obes Res 2003; 11:1048-1054.

33. Jorge M, de Oliveira V, Resende N, Paraiso L, Calixto A, Diniz A, Resende E, et al. The effects of aerobic, resistance, and combined exercise African Health Sciences Vol 13 Issue 4 December 2013 
on metabolic control, inflammatory markers, adipocytokines, and muscle insulin signaling in patients with type 2 diabetes mellitus. Metabolism 2011; 60: 1244-1252.

34. Smith J, Dykes R, Douglas J, Krishnaswamy G, Berk S. Long-term exercise and atherogenic activity of blood mononuclear cells in persons at risk of developing ischemic heart disease. $J A M A$ 1999; 281:1722-7.

35. Stewart K. Role of exercise training on cardiovascular disease in persons who have type 2 diabetes and hypertension. Cradiol Clin 2004; 22: $569-86$

36. Miller J, Pratley R, Goldberg A, Gordon P., Rubin M, Treuth M. Strength training increases insulin action in healthy 50- to 65-year-old men. J Appl Physiol 1994; 77: 1122-1127.

37. Ryan A, Pratley R, Elahi D, Goldberg A. Changes in plasma leptin and insulin action with resistive training in postmenopausal women. Int J Obes Relat Metab Disord 2000; 24: 27-32.

38. Zachwieja J, Toffolo G, Cobelli C, Bier D, Yarasheski K. Resistance exercise and growth hormone administration in older men: effects on insulin sensitivity and secretion during a stablelabel intravenous glucose tolerance test. Metabolism 1996; 45:254-60.

39. Hansen D, Dendale P, Jonkers RA. Continuous low- to moderate-intensity exercise training is as effective as moderate- to high-intensity exercise training at lowering blood $\mathrm{HbA}(1 \mathrm{c})$ in obese type 2 diabetes patients. Diabetologia 2009; 52: 1789-1797.

40. Dunstan DW, Daly RM, Owen N. High-intensity resistance training improves glycemic control in older patients with type 2-diabetes. Diabetes Care 2002; 25:1729-1736. 